\title{
La traduction automatique comme outil d'aide à la rédaction scientifique en anglais langue seconde : résultats d'une étude exploratoire sur la qualité linguistique
}

Using machine translation for academic writing in English as a second language: results of an exploratory study on linguistic quality

Marie-Josée Goulet, Michel Simard, Carla Parra Escartín et Sharon O'Brien

\section{(2) OpenEdition}

Journals

Édition électronique

URL : http://journals.openedition.org/asp/5045

DOI : 10.4000/asp.5045

ISSN : 2108-6354

\section{Éditeur}

Groupe d'étude et de recherche en anglais de spécialité

Édition imprimée

Date de publication : 1 novembre 2017

Pagination : $5-28$

ISSN : 1246-8185

Référence électronique

Marie-Josée Goulet, Michel Simard, Carla Parra Escartín et Sharon O’Brien, « La traduction

automatique comme outil d'aide à la rédaction scientifique en anglais langue seconde : résultats d'une étude exploratoire sur la qualité linguistique », ASp [En ligne], 72 | 2017, mis en ligne le 01 novembre 2018, consulté le 01 novembre 2020. URL : http://journals.openedition.org/asp/5045 ; DOI : https:// doi.org/10.4000/asp.5045

Ce document a été généré automatiquement le 1 novembre 2020.

Tous droits réservés 


\section{La traduction automatique comme outil d'aide à la rédaction} scientifique en anglais langue seconde : résultats d'une étude exploratoire sur la qualité linguistique

Using machine translation for academic writing in English as a second language: results of an exploratory study on linguistic quality

Marie-Josée Goulet, Michel Simard, Carla Parra Escartín et Sharon O’Brien

\section{Introduction}

1 Nous nous intéressons dans cet article à l'utilisation de la traduction automatique (TA) pour assister la rédaction de textes en anglais de spécialité ${ }^{1}$. Considérant que l'anglais est la langue dominante des publications savantes (Graham et alii 2011; Bennett 2013, 2014a, 2014b, 2015 ; Breuer 2015), il est raisonnable de penser que la TA pourrait être utile pour les chercheurs qui n'ont pas l'anglais comme langue maternelle et qui sont amenés à rédiger des articles en anglais. Plusieurs études suggèrent que certaines personnes qui rédigent en anglais langue seconde ou étrangère se sentent désavantagées (Hanauer \& Englander 2011) ou sont moins productives (Van Waes \& Leijten 2015). Dans ce contexte, nous croyons que la TA pourrait être bénéfique, dans la mesure, bien entendu, où son utilisation ne nuit pas à la qualité du texte.

2 Au cours des dernières années, la TA statistique a connu des progrès significatifs, notamment en termes de coût, de rapidité et de qualité (Bahadur \& Chaudan 2014 ; O'Brien \& Simard 2014 ; Ebrahim et alii 2015). Les avancées sont telles qu'un nombre 
grandissant d'entreprises de services langagiers ont recours à la TA (DePalma et alii 2013). La TA n'est toutefois pas l'apanage des entreprises ou des traducteurs professionnels; elle est également accessible au grand public. L'on n'a qu'à penser à Microsoft Translator intégré au traitement de texte Word, à Google Translate ou encore à BabelFish, ces deux derniers étant offerts gratuitement sur le web. L'utilisation des technologies de TA par des particuliers, c'est-à-dire des personnes qui s'adonnent à des activités de traduction, mais qui n'ont pas été formées pour le faire, commence à être documentée (Hu et alii 2011 ; Koehn 2010 ; Mitchell et alii 2014), mais aucune étude sur son utilité dans le contexte de la rédaction scientifique n'a encore été publiée.

Dans cet article, nous explorons la question de l'utilité de la TA comme outil d'aide à la rédaction d'articles scientifiques en anglais de spécialité ${ }^{2}$. Comme première tentative de réponse, nous abordons le sujet sous l'angle de la qualité linguistique, ce qui comprend la morphologie, la sémantique, le style, la syntaxe, la typographie et la ponctuation. Plus précisément, nous analysons dans cette étude exploratoire les modifications linguistiques effectuées par une réviseure professionnelle dans des résumés rédigés par des chercheurs de divers domaines. Les résumés ont la particularité d'être composés d'une partie rédigée directement en anglais langue seconde (ALS) et d'une partie rédigée en langue maternelle, traduite automatiquement et post-éditée ${ }^{3}$. La qualité linguistique sera déterminée par le nombre de modifications linguistiques, une mesure inspirée du Human-targeted Translation Edit Rate (HTER), lequel est couramment utilisé pour mesurer la qualité de textes traduits automatiquement (Snover et alii 2006). Plus précisément, le HTER correspond au nombre de mots qui doivent être modifiés afin de rendre un texte équivalent à sa version définitive postéditée. Plus ce nombre est petit, plus la qualité du texte évalué est considérée bonne.

Dans la section suivante, nous présentons l'état de la question sur la rédaction scientifique en ALS et sur l'utilisation de la TA dans des tâches de rédaction en langue seconde. Cet état de la question se conclut par la problématique et la formulation des questions de recherche. Dans la section 3, nous décrivons la méthodologie déployée afin de répondre à nos questions de recherche. Dans la section 4 , nous présentons les résultats de l'analyse, de même qu'une discussion. Enfin, nous concluons dans la section 5 .

\section{2. État de la question et problématique}

5 De manière générale, la rédaction en langue seconde constitue un sujet de recherche relativement bien documenté. Dans cette section, nous présentons les études antérieures pertinentes par rapport à l'utilisation de la TA pour la rédaction d'articles scientifiques en ALS. La décision de publier un article en ALS plutôt que dans sa langue maternelle serait influencée par divers facteurs, par exemple le désir de diffuser ses résultats à l'international (Burgess et alii 2014; Martìn et alii 2014) et de se faire reconnaître par ses pairs (López-Navarro et alii 2015). La discipline du chercheur serait également un facteur déterminant dans cette décision. Une étude effectuée à l'Université Santiago de Compostela a démontré que les chercheurs issus des sciences expérimentales et de la santé étaient plus nombreux à publier en ALS, tandis que les chercheurs issus des sciences humaines et sociales étaient moins nombreux à prendre cette décision (Fernández Polo \& Cal Varela 2009). Cette conclusion s'appuie sur une analyse de 213 questionnaires. Les résultats de Sally Burgess et alii (2014) vont dans le 
même sens. Selon les 1717 questionnaires analysés, le désir de publier en anglais est plus grand pour les chercheurs en psychologie que pour ceux en histoire.

6 Cette décision de publier en ALS ne serait pas sans conséquence. En effet, comme l'ont souligné John R. Benfield et Christine B. Feak (2006), les articles rédigés en ALS et soumis à des revues scientifiques requièrent généralement plus de modifications visant à améliorer la clarté que les articles rédigés par des locuteurs natifs de l'anglais. Cette observation s'appliquait au domaine médical, mais il est aisé d'admettre qu'elle puisse être valide dans plusieurs autres domaines. En effet, plusieurs chercheurs font appel à des professionnels de la langue pour réviser leurs articles (Lillis \& Curry 2010). Toutefois, cette solution entraîne des coûts, aussi bien en temps qu'en argent. De plus, il peut s'avérer ardu de trouver un réviseur professionnel qui possède une expertise dans tel sujet scientifique.

7 En outre, la rédaction scientifique en ALS présente des défis. David I. Hanauer et Karen Englander (2011) ont abordé cette question en tentant de quantifier le «fardeau » que représente le fait de rédiger en ALS. À partir des perceptions de 141 chercheurs mexicains, recueillies dans un court sondage, les auteurs ont montré que la rédaction en ALS était associée à une plus grande anxiété et à une moins grande satisfaction une fois l'article terminé, en comparaison avec la rédaction en langue maternelle. Notons que ces différences sont significatives.

8 Pour des mesures plus objectives des défis que pose la rédaction en langue seconde, il faut se tourner vers les études sur des étudiants universitaires. Par exemple, Luuk Van Waes et Mariëlle Leijten (2015) ont observé 68 étudiants pendant qu'ils rédigeaient. À partir de mesures telles que le nombre de mots tapés par minute, le nombre de pauses requises et la durée des pauses, ces auteurs ont découvert que les étudiants étaient moins productifs lorsqu'ils rédigeaient en langue seconde (anglais, allemand, espagnol ou français), en comparaison avec leur langue maternelle (hollandais). Pour sa part, Esther Odilia Breuer (2015) a analysé 40 dissertations d'étudiants universitaires afin de mesurer l'effet de diverses techniques d'écriture. L'une des conclusions est que la technique de l'écriture libre (freewriting) provoque un effet moins exaltant en langue seconde (anglais) qu'en langue maternelle (allemand), ce qui pourrait indiquer une moins grande facilité à réfléchir lorsqu'on rédige dans une langue seconde, en comparaison avec la rédaction en langue maternelle.

9 Le portrait que nous venons de dresser est sans équivoque: la rédaction en ALS présente des défis pour les chercheurs qui choisissent cette option. Les résultats des études antérieures sont autant de justifications pour chercher de nouvelles façons d'appuyer la rédaction d'articles scientifiques en ALS. La méthode que nous proposons consiste à rédiger un texte scientifique en langue maternelle, à le faire traduire automatiquement puis à le post-éditer. Notons que le terme post-édition est généralement réservé afin de désigner la révision d'un texte traduit automatiquement. L'activité de révision, quant à elle, consiste à relire un texte dans le but d'en améliorer la qualité et s'applique normalement à des textes rédigés ou traduits par des humains. Ainsi, la post-édition et la révision partagent le même objectif - améliorer la qualité d'un texte -, mais ne s'opèrent pas sur le même type de texte.

La méthode que nous proposons est inspirée des recherches sur l'utilisation de la TA dans le domaine de l'apprentissage des langues secondes. Mentionnons en premier lieu l'étude d'Ana Niño (2008), qui impliquait 32 apprenants avancés de l'espagnol et locuteurs natifs de l'anglais. Pour les participants du groupe expérimental, l'épreuve 
consistait à post-éditer un texte traduit en espagnol par le logiciel Systran Professional Standard 3.0. Au besoin, ils pouvaient consulter la version originale anglaise. Notons que les étudiants de ce groupe avaient préalablement assisté à dix séances de formation sur la post-édition. Pour leur part, les participants du groupe témoin devaient traduire les mêmes textes sources, de l'anglais vers l'espagnol. Les textes issus des deux groupes ont ensuite été corrigés manuellement ${ }^{4}$ et chaque correction a été annotée suivant la typologie élaborée par d'A. Niño (2008). Il faut entendre ici la correction comme une activité consistant à évaluer la qualité d'un texte produit par un étudiant dans un contexte d'apprentissage d'une langue. Selon les résultats de l'analyse, le nombre d'erreurs dans les textes post-édités est inférieur au nombre d'erreurs dans les textes traduits, et ce, pour tous les aspects de la typologie (lexique, grammaire, discours et orthographe). De plus, les erreurs présentes dans les textes post-édités ne sont pas très différentes de celles que l'on retrouve dans les textes traduits par les étudiants. Selon A. Niño (2008), ces résultats suggèrent que des apprenants avancés sont aptes à postéditer des textes traduits automatiquement dans leur langue seconde de manière à les rendre compréhensibles, du moins aussi compréhensibles que des textes traduits par des apprenants du même niveau.

11 Les résultats vont dans le même sens dans l'étude d'Ignacio García et de María Isabel Pena (2011), à laquelle ont participé seize personnes ayant l'anglais comme langue maternelle et apprenant l'espagnol. Dans cette expérience, chaque participant devait rédiger deux courts textes : un premier directement en langue maternelle (anglais) et un second en espagnol. Pour la première tâche, les participants ont utilisé l'interface Tradukka ${ }^{5}$, une application gratuite qui permet à l'utilisateur de voir la traduction simultanée (fenêtre de droite) du texte qu'il est en train de taper (fenêtre de gauche). Notons que, selon les informations fournies dans l'article, Tradukka utilisait la technologie de Google Translate en 2011. Pendant l'expérience, les données provenant du clavier et du curseur ont été enregistrées. Ensuite, les textes rédigés par les participants ont été envoyés à deux enseignants. Ces derniers devaient attribuer une note globale pour chaque texte, en tenant compte de divers critères: erreurs grammaticales et lexicales, vocabulaire, temps verbaux, atteinte de l'objectif de communication et lisibilité. L'analyse révèle tout d'abord que les débutants ont écrit plus de mots avec l'aide de la TA. De plus, les textes écrits avec l'aide de la TA ont obtenu des notes moyennes plus élevées que ceux écrits directement en espagnol langue seconde. Selon I. García et M. I. Pena (2011), ces résultats indiquent que la TA aide l'apprenant débutant à communiquer plus et mieux dans la langue seconde. Dans la deuxième partie de l'analyse, les auteurs montrent que les apprenants effectuent plus de pauses lorsqu'ils rédigent directement en langue seconde que lorsqu'ils utilisent la TA. Ces résultats pourraient indiquer, toujours selon I. García et M. I. Pena (2011), que la rédaction en langue seconde requiert plus d'effort cognitif que la rédaction en langue maternelle assistée par la TA. Autre point intéressant : le nombre de modifications effectuées par les participants dans leurs propres textes est comparable dans les deux situations. Toutefois, l'analyse des modifications suggère qu'il existe une relation entre les apprenants de niveau plus avancé et un plus grand nombre de modifications valides.

En dernier lieu, Errol Marinus O'Neill (2012) a étudié les effets quantitatifs et qualitatifs de l'utilisation de la TA sur l'écriture en langue seconde. L'expérience, qui incluait 32 étudiants universitaires ayant l'anglais comme langue maternelle et apprenant le français, comprenait trois scénarios différents: dans le groupe 1, les participants 
étaient autorisés à utiliser le moteur en ligne Free Translation ${ }^{6}$ après avoir assisté à une formation; dans le groupe 2, les participants étaient autorisés à utiliser le moteur de traduction, mais n'avaient pas reçu de formation préalable ; dans le groupe 3 (groupe témoin), les participants n'avaient pas suivi de formation et n'étaient pas autorisés à utiliser le moteur de traduction. Chaque participant devait rédiger deux courts textes, qui étaient ensuite notés par des professeurs de français langue seconde, en fonction des critères suivants: intelligibilité, contenu, orthographe, syntaxe, grammaire et vocabulaire. Dans une des deux tâches, les notes accordées par les juges sont plus élevées pour les textes du groupe 1, en comparaison avec les textes du groupe témoin. De plus, dans les deux tâches, les groupes 1 et 2 ont largement surpassé le groupe témoin dans quatre des six aspects évalués.

13 Considérant l'ensemble des études antérieures présentées dans cette section, il nous apparaît clair que l'utilisation de la TA pourrait procurer certains avantages dans le contexte de la rédaction scientifique en ALS. De plus, les résultats combinés des études antérieures nous permettent de poser l'hypothèse que les chercheurs seraient aptes à post-éditer des textes traduits automatiquement en anglais, de manière à les rendre de qualité acceptable. Bien entendu, cette hypothèse soulève la question de savoir comment mesurer la qualité. Dans les études précédentes (Breuer 2015 ; O’Neill 2012 ; García \& Pena 2011; Niño 2008), la qualité était déterminée par les notes des juges externes. Cette manière de concevoir la qualité est tout à fait pertinente dans le contexte de l'apprentissage d'une langue seconde, où les textes des élèves sont régulièrement évalués par leurs enseignants. Dans cet article, nous adoptons une perspective légèrement différente, à savoir que la qualité linguistique des textes sera déterminée à partir du nombre de modifications effectuées par une réviseure professionnelle. Cette mise en situation est tout à fait réaliste, puisque les chercheurs qui rédigent des articles scientifiques en ALS font parfois appel à des professionnels de la langue pour faire réviser leurs textes (Lillis \& Curry 2010).

L'on aura remarqué, par ailleurs, que les études antérieures combinent normalement différents critères afin de mesurer la qualité des textes. Certains critères, comme l'orthographe et la grammaire, ont trait à la qualité linguistique tandis que d'autres, comme la réalisation de l'objectif de communication, concernent le contenu. Dans cet article, nous nous concentrons sur la qualité linguistique des textes, plus précisément sur la morphologie, la sémantique, la syntaxe, le style et la ponctuation. Nous sommes conscients de l'importance d'évaluer la qualité du contenu des textes, mais nous avons choisi de miser sur la profondeur de l'analyse linguistique. Nous sommes également d'avis que d'autres aspects de la méthode proposée devraient être étudiés, notamment l'effort cognitif déployé par les rédacteurs et le temps requis pour effectuer la révision des textes, mais que ces sujets devront être abordés dans une recherche ultérieure.

$\mathrm{Au}$ terme de cette réflexion, nous sommes en mesure de formuler nos questions de recherche. Premièrement, nous cherchons à savoir si les articles scientifiques rédigés avec l'aide de la TA engendrent plus ou moins de modifications linguistiques de la part d'une réviseure professionnelle que les articles rédigés directement en ALS. Deuxièmement, les modifications sont-elles du même type dans les deux situations? C'est ainsi que nous pourrons déterminer si l'utilisation de la TA entraîne un effet sur la qualité linguistique et, le cas échéant, si cette différence peut être attribuée à un aspect en particulier. 


\section{Méthodologie}

\subsection{Recrutement des participants}

16 Notre recherche requérait de recruter des chercheurs qui ont à rédiger des articles en anglais de spécialité. Pour ce faire, nous avons envoyé des courriels à des collègues de nos institutions respectives, de même qu'à des collègues d'autres universités et centres de recherche. Le message envoyé décrivait brièvement l'objectif de la recherche et précisait que nous étions à la recherche de doctorants ou de chercheurs en début de carrière qui doivent rédiger des textes en anglais, mais pour qui l'anglais n'est pas la langue maternelle. Le message précisait en outre que l'expérience durerait environ quatre heures, réparties sur six semaines, et que chaque participant qui aurait complété toutes les étapes de la recherche se verrait offrir un chèque-cadeau d'une valeur de 70 euros, valable auprès d'une boutique en ligne réputée. La rétribution des participants avait pour but d'augmenter nos chances de recrutement.

Le message de recrutement incluait un lien qui menait à un court questionnaire, que pouvaient remplir les personnes potentiellement intéressées. Les réponses à ce questionnaire nous ont permis de vérifier l'admissibilité des participants, notamment en regard de la langue maternelle qui ne devait pas être l'anglais. De plus, le questionnaire demandait aux répondants d'évaluer leur niveau d'anglais, selon l'échelle suivante : débutant, intermédiaire, avancé, expert. Une description sommaire était fournie pour chaque niveau. Cette question visait à identifier les candidats pour lesquels la TA était susceptible d'être utile, à savoir des locuteurs intermédiaires ou avancés de l'ALS. Par ailleurs, l'auto-évaluation du niveau de compétence en anglais est une méthode relativement répandue dans notre domaine (voir, entre autres, Burgess et alii 2014 ; Fernández Polo \& Cal Varela 2009). Enfin, les participants devaient choisir parmi l'une des trois tâches suivantes :

- Rédiger un résumé de 500 mots sur sa recherche doctorale ;

- Rédiger un résumé de 500 mots sur un article en préparation;

- Rédiger un résumé de 500 mots sur une proposition de recherche en préparation.

$18 \mathrm{Au}$ terme de notre campagne de recrutement, 14 personnes avaient accepté de participer à notre recherche. De ce nombre, trois n'ont pas achevé toutes les étapes et une a été disqualifiée, faute d'avoir suivi correctement les consignes. Le nombre final de participants est ainsi de dix. Les langues maternelles des participants sont l'allemand (1), l'arabe (1), le chinois (1), l'espagnol (2), le français (4) et le roumain (1). Ils travaillent dans des domaines de recherche variés : biotechnologie (3), chimie (1), géologie (1), génie appliqué (1), marketing (1), psychologie (2) et sciences sociales (1). Sept sont des doctorants, un est post-doctorant, un est assistant de recherche et un est professeur ${ }^{7}$. Par ailleurs, la moitié des participants (5) possède une expérience considérable dans la rédaction d'articles scientifiques en anglais (entre 8 et 12 articles publiés), tandis que l'autre moitié (5) a peu d'expérience (1 ou 0 article publié).

Comme nous l'avons mentionné précédemment, les participants devaient évaluer leur niveau de compétence en ALS avant de commencer la rédaction. Six d'entre eux ont qualifié leur niveau d'intermédiaire, trois d'avancé et un entre intermédiaire et avancé. Aucun participant ne s'est décrit comme un débutant ou un expert. Notons en outre que près de la moitié des participants ( 4 sur 10) a affirmé avoir déjà utilisé la TA afin de produire un texte scientifique en anglais. 
Quant à la tâche à effectuer, quatre participants ont choisi de rédiger un résumé de leur recherche doctorale, deux ont opté pour un résumé d'article et quatre ont rédigé une proposition de recherche.

\subsection{Rédaction des résumés}

Le processus de production des textes s'est déroulé en deux temps. À chacune des étapes, les participants ont reçu des consignes détaillées, par courriel. Au cours de la première phase, les participants devaient rédiger un premier jet de leur résumé. Ce résumé devait être divisé en deux parties et structuré selon les consignes suivantes, lesquelles s'inspirent des recommandations de Melida J. Ikes et Stephen F. Gambescia (2011). La partie A du résumé devait compter environ 250 mots et contenir ces éléments : titre indiquant clairement le sujet du texte, contexte de la recherche et problématique. La partie $B$ du résumé devait compter elle aussi environ 250 mots et contenir les éléments suivants : méthodologie, aperçu et implications des résultats.

Les participants devaient rédiger une partie du résumé en langue maternelle et une partie en anglais. Nous avons pris soin d'alterner d'un participant à l'autre, de manière à éviter que l'ordre dans lequel les langues étaient utilisées ne vienne biaiser les résultats. Au final, six participants ont rédigé la partie $\mathrm{A}$ en anglais et la partie $\mathrm{B}$ dans leur langue maternelle, tandis que les quatre autres ont fait l'inverse. Par ailleurs, comme précisé plus haut, les participants pouvaient choisir le type de résumé qu'ils rédigeraient. Cette mesure avait pour but d'accommoder les participants et, ainsi, de favoriser le recrutement.

La méthode de rédaction proposée, si elle n'est pas naturelle, représentait le meilleur compromis afin d'obtenir des résumés comparables. Idéalement, nous aurions demandé à chaque participant de produire deux résumés complets, l'un en langue maternelle et l'autre en ALS. Dans ce cas, toutefois, il aurait fallu que les résumés portent sur des sujets différents, ce qui aurait exclu plusieurs participants potentiels. De plus, cette manière de procéder aurait allongé la durée de l'expérience pour les participants, ce qui aurait davantage accentué les difficultés de recrutement.

24 Les participants n'avaient pas le droit de consulter des collègues ou des amis. Les participants avaient la permission d'utiliser des outils informatiques, mais pas de logiciel de traduction automatique. Grâce aux informations fournies dans le questionnaire qui concluait leur participation, nous savons que les chercheurs de notre expérience ont utilisé des correcteurs (ex.: Word), des dictionnaires de synonymes (ex. : Word), des dictionnaires unilingues (ex. : Oxford) et des dictionnaires bilingues (ex. : Grand dictionnaire terminologique ${ }^{8}$ et WordReference ${ }^{9}$ ). Aucun autre outil n'a été mentionné.

25 Cette première étape terminée, les participants nous ont transmis leur résumé par courriel.

\subsection{Traduction, post-édition et révision des résumés}

La partie des résumés rédigée en langue maternelle a été traduite en anglais par Google Translate ${ }^{10}$. Cette étape de l'expérimentation ayant eu lieu au cours de l'hiver 2015-2016, les traductions ont vraisemblablement été produites avec la TA statistique de Google, puisque la technologie dite «neuronale » n'a été déployée qu'en novembre 
2016 (Turovsky 2016). Notre choix s'est arrêté sur Google Translate, car au moment de conduire l'étude, il offrait la plus grande variété de paires de langues, en comparaison avec Systran $^{11}$ et Reverso ${ }^{12}$, par exemple. De plus, les quelques essais informels que nous avons effectués avec d'autres moteurs de traduction en ligne suggéraient que Google Translate produisait généralement des traductions de qualité supérieure à celles de ses concurrents, par exemple Bing ${ }^{13}$.

La TA a permis d'obtenir une deuxième version du résumé, entièrement en anglais. Puis, nous avons renvoyé les résumés aux participants, en précisant que la partie qu'ils avaient rédigée en langue maternelle avait été traduite par un logiciel. Lors de cette deuxième phase, les participants devaient post-éditer leur résumé, en suivant les consignes suivantes :

- Veiller à ce que les phrases soient grammaticalement correctes ;

- Vérifier la traduction des termes spécialisés ;

- S'assurer qu'aucune information n'ait été ajoutée, élidée ou non traduite ;

- Appliquer les règles de base pour l'orthographe et la ponctuation.

Nous avons formulé ces consignes à partir des principes fournis sur le site du consortium Translation Automation User Society (TAUS) (Massardo et alii 2010), qui sont destinés aux traducteurs professionnels et aux industries de la langue. Nos consignes n'impliquaient pas que les participants vérifient l'adéquation de la traduction avec le texte source. Nous avons plutôt misé sur le fait que les participants connaissaient leur texte. S'ils le souhaitaient, les participants pouvaient également apporter des modifications à la partie qu'ils avaient initialement rédigée en anglais. Après nous avoir renvoyé leur résumé, les participants étaient invités à remplir un court questionnaire sur leur expérience.

Par la suite, une réviseure professionnelle a été engagée pour la révision des résumés. La réviseure est une locutrice native de l'anglais et a plusieurs années d'expérience en traduction et révision. Afin de la guider adéquatement dans sa tâche, nous lui avons expliqué que le résultat attendu était que les textes se lisent suffisamment bien pour être soumis à un colloque scientifique, par exemple. Plus précisément, la réviseure professionnelle devait se concentrer sur la grammaire, l'orthographe, la ponctuation, la syntaxe et le style, en précisant pour ce dernier point que seuls les problèmes majeurs devaient faire l'objet d'une modification. Nous lui avons également demandé d'activer le suivi des modifications dans son logiciel de traitement de texte.

Bien entendu, la réviseure ne savait pas que les résumés avaient été rédigés par des locuteurs non natifs de l'anglais. Elle ne savait pas, non plus, qu'une partie des résumés (la première ou la deuxième moitié selon le cas) avait été traduite automatiquement en anglais puis post-éditée par l'auteur. Les résumés fournis à la réviseure professionnelle étaient exempts de toute trace du processus méthodologique que nous venons de décrire. La figure 1 représente le processus de rédaction, de post-édition et de révision des résumés scientifiques. La partie supérieure de la figure représente la rédaction directe en ALS ou rédaction non assistée, tandis que la partie inférieure représente la rédaction assistée par la TA. 
Figure 1. Processus de rédaction, de post-édition et de révision des résumés

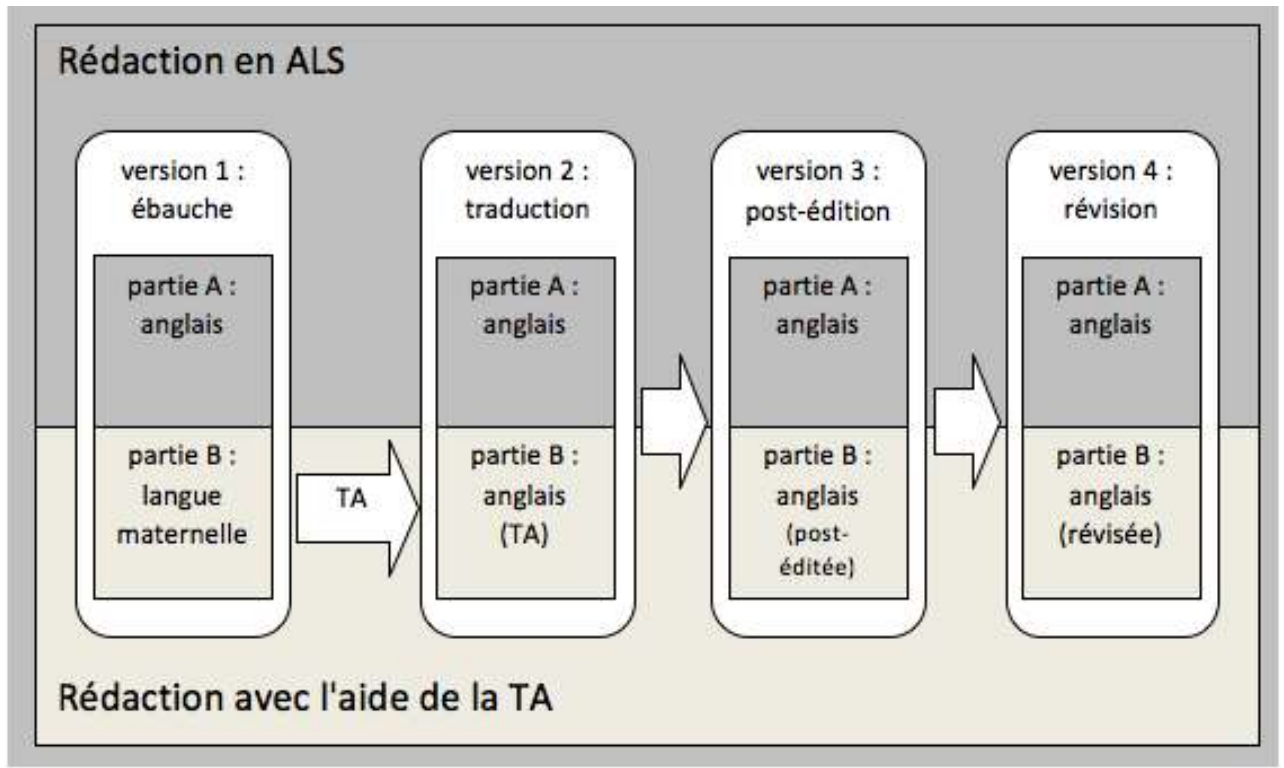

\subsection{Annotation des modifications linguistiques} linguistiques effectuées par une réviseure professionnelle dans les résumés produits par des chercheurs de divers domaines. Grâce à l'analyse des modifications effectuées par la réviseure professionnelle, nous serons en mesure de déterminer si l'utilisation de la TA engendre un plus grand nombre ou des types différents de modifications linguistiques, en comparaison avec la rédaction directe en ALS.

Il existe plusieurs typologies de post-édition et de révision linguistique, utilisées dans différents contextes : textes traduits par des humains (Mossop 2014), textes traduits automatiquement et post-édités (Niño 2008; Mitchell et alii 2014; Sánchez-Gijón \& Torres-Hostench 2014), textes rédigés en langue étrangère (Breuer 2015) et textes rédigés en langue maternelle (Laflamme 2009). Nous avons retenu la typologie de Caroline Laflamme (2009), qui est la plus cohérente avec la directive que nous avons donnée à la réviseure professionnelle. Par ailleurs, la typologie de C. Laflamme (2009) est représentative de la pratique réelle des réviseurs professionnels, puisqu'elle résulte d'une étude dans laquelle on a filmé six professionnels pendant qu'ils révisaient des textes rédigés en langue maternelle. Le tableau 1 présente la typologie de C. Laflamme (2009). 
Tableau 1. Typologie de C. Laflamme (2009) pour les modifications effectuées en révision professionnelle

\begin{tabular}{|c|c|c|}
\hline $\begin{array}{l}\text { Opérations } \\
\text { - Ajout } \\
\text { - Déplacement } \\
\text { - Remplacement } \\
\text { - Suppression }\end{array}$ & $\begin{array}{l}\text { Unités linguistiques } \\
\text { - Adjectif } \\
\text { - Adverbe } \\
\text { - Conjonction } \\
\text { - Déterminant } \\
\text { - Nom } \\
\text { - Préposition } \\
\text { - Pronom } \\
\text { - Verbe }\end{array}$ & $\begin{array}{l}\text { Dimensions linguistiques } \\
\text { - Morphologie } \\
\text { - Sémantique } \\
\text { - Style } \\
\text { - Syntaxe } \\
\text { - Typographie }\end{array}$ \\
\hline
\end{tabular}

La typologie retenue permet d'annoter les modifications selon trois aspects: le type d'opération, le type d'unité et la dimension linguistique. Nous avons dû, toutefois, apporter de légers changements à cette typologie. D'abord, comme cette typologie porte uniquement sur les modifications lexicales, c'est-à-dire celles qui affectent les mots, nous avons ajouté la dimension "ponctuation» qui comprend les signes de ponctuation. De plus, lorsqu'une modification touchait une suite de mots, nous avons considéré la suite de mots comme une unité, plutôt que d'annoter chaque mot de la suite. Nous avons utilisé la catégorie « syntagme » pour les suites de mots. Le tableau 2 présente la typologie amendée, avec les ajouts en gras.

Tableau 2. Typologie amendée de C. Laflamme (2009) pour les modifications effectuées en révision professionnelle

\begin{tabular}{|c|c|c|}
\hline $\begin{array}{l}\text { Opérations } \\
\qquad \begin{array}{l}\text { - Ajout } \\
\text { • Déplacement } \\
\text { • Remplacement } \\
\text { - Suppression }\end{array}\end{array}$ & $\begin{array}{l}\text { Unités linguistiques } \\
\text { - Adjectif } \\
\text { - Adverbe } \\
\text { - Conjonction } \\
\text { - Déterminant } \\
\text { - Nom } \\
\text { - Préposition } \\
\text { - Pronom } \\
\text { - Verbe } \\
\text { - Syntagme } \\
\text { - Signe de ponctuation }\end{array}$ & $\begin{array}{l}\text { Dimensions linguistiques } \\
\text { - Morphologie } \\
\text { - Sémantique } \\
\text { - Style } \\
\text { - Syntaxe } \\
\text { - Typographie } \\
\text { - Ponctuation }\end{array}$ \\
\hline
\end{tabular}

Nous devons également fournir quelques précisions sur la manière dont nous avons appliqué cette typologie. La catégorie "unités linguistiques» s'applique à l'unité d'origine. Dans la grande majorité des cas, une unité a été remplacée par une unité du même type, par exemple un nom par un nom. Dans d'autres cas, en nombre toutefois moins important, une unité a été remplacée par une unité d'un autre type, par exemple une préposition par un verbe. Dans ces cas, nous avons annoté la modification en fonction du mot affecté par la modification, et non en fonction du résultat de la modification. Par ailleurs, nous avons quelquefois observé une modification qui causait une autre modification. Dans ce cas, nous avons traité la deuxième modification (effet) comme faisant partie de la première modification (cause). 
Grâce à l'outil "suivi des modifications" du traitement de texte, nous avons pu prendre connaissance de tous les changements effectués par la réviseure professionnelle. Chaque modification a été annotée à l'aide de la typologie amendée de C. Laflamme (2009) (voir tableau 2). Voici quelques exemples d'extraits annotés, où les modifications sont en gras.

Tableau 3. Exemples d'extraits annotés

\begin{tabular}{|c|c|c|}
\hline $\begin{array}{l}\text { Identifiant du } \\
\text { participant }\end{array}$ & Extrait du résumé & Annotation \\
\hline P11 & $\begin{array}{l}\text { Data were was collected through focus groups and semi- } \\
\text { structured interviews... }\end{array}$ & $\begin{array}{l}\text { remplacement } \\
\text { verbe } \\
\text { morphologie }\end{array}$ \\
\hline P14 & $\begin{array}{l}\text { This article examines alternatives to bureaucracy in the context } \\
\text { of the new public management. }\end{array}$ & $\begin{array}{l}\text { suppression } \\
\text { déterminant } \\
\text { syntaxe }\end{array}$ \\
\hline P1 & $\begin{array}{l}\text { To measure the temperature-induced shrinking effect, a digital } \\
\text { microscope was used to take photos of the hydrogels in } 10^{\circ} \mathrm{C} \\
\text { intervals... }\end{array}$ & $\begin{array}{l}\text { ajout } \\
\text { virgule } \\
\text { ponctuation }\end{array}$ \\
\hline P8 & $\begin{array}{l}\text { No adverse events reactions were reported by our participants } \\
\text { during or following the immersions. }\end{array}$ & $\begin{array}{l}\text { remplacement } \\
\text { nom } \\
\text { sémantique }\end{array}$ \\
\hline P2 & $\begin{array}{l}\text { few researches have studied the relationship between the } \\
\text { control parameters of the laser processing machines and } \\
\text { microfluidic characteristics }\end{array}$ & $\begin{array}{l}\text { ajout } \\
\text { verbe } \\
\text { style }\end{array}$ \\
\hline P5 & $\begin{array}{l}\text { As the molecular mechanisms of the metabolic shift phenomenon } \\
\text { known as the "Warbrug Warburg effect" are currently not well } \\
\text { known... }\end{array}$ & $\begin{array}{l}\text { remplacement } \\
\text { nom } \\
\text { typographie }\end{array}$ \\
\hline
\end{tabular}

Pour conclure sur la méthodologie, l'annotation des modifications a été effectuée par deux des auteurs. Dans un premier temps, elles ont annoté un résumé (P2), chacune de leur côté. Puis, elles ont discuté des cas où elles étaient en désaccord. Après cette discussion, elles ont refait le même processus avec un deuxième résumé (P4). Dans ce cas, les désaccords étaient moins nombreux. Les huit autres résumés (quatre chacune) ont ensuite été annotés et chacune a vérifié les annotations de l'autre. Chaque cas de désaccord a été discuté entre les auteures afin d'en arriver à une annotation finale. Lors de cette vérification interjuges, les annotations concordaient dans des proportions très satisfaisantes : dans $95 \%$ des cas pour les types d'opérations, dans $92 \%$ des cas pour les unités linguistiques et dans $82 \%$ des cas pour les dimensions linguistiques. Ces résultats indiquent qu'après discussion entre les deux annotatrices, la typologie de C. Laflamme (2009) a pu être appliquée de manière précise et systématique. 


\section{Résultats de l'analyse des modifications linguistiques} linguistiques effectuées par la réviseure professionnelle. La figure 2 présente les statistiques générales.

Figure 2. Statistiques générales

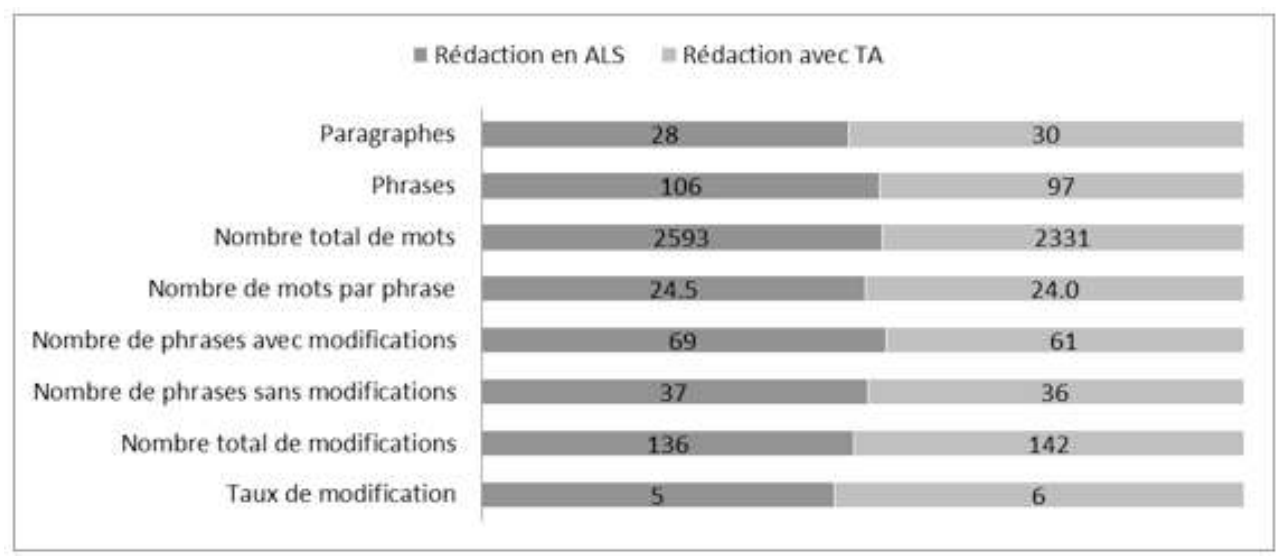

Comme on peut le voir dans cette figure, le nombre de mots est de 2593 pour la rédaction en ALS et de 2331 pour la rédaction avec la TA, ce qui donne un total de 4924 mots. Dans les parties rédigées en ALS, le nombre de modifications linguistiques est de 136, alors qu'il est de 142 dans les parties où la TA a été utilisée. La dernière ligne de la figure 2 fournit le taux de modification, c'est-à-dire le nombre de modifications par 100 mots. Ce dernier est de 5 dans les textes rédigés en ALS et de 6 dans les textes traduits automatiquement et post-édités. La réponse à notre première question de recherche est donc que les textes produits avec l'aide de la TA ont engendré légèrement plus de modifications linguistiques de la part de la réviseure professionnelle. Toutefois, compte tenu de la taille modeste de notre échantillon, il n'y a pas lieu de croire que cette différence soit significative. Nous concluons que la qualité linguistique est sensiblement la même dans les textes rédigés en ALS et dans ceux produits avec l'aide de la TA.

L'annotation des modifications effectuées par la réviseure professionnelle nous permet d'examiner plus en détail la distribution des modifications dans les deux situations, relativement aux trois aspects suivants:type d'opération effectuée, type d'unité linguistique visée et dimension linguistique. C'est ce que présentent respectivement les figures 3,4 et 5 . 
Figure 3. Distribution des modifications par type d'opération

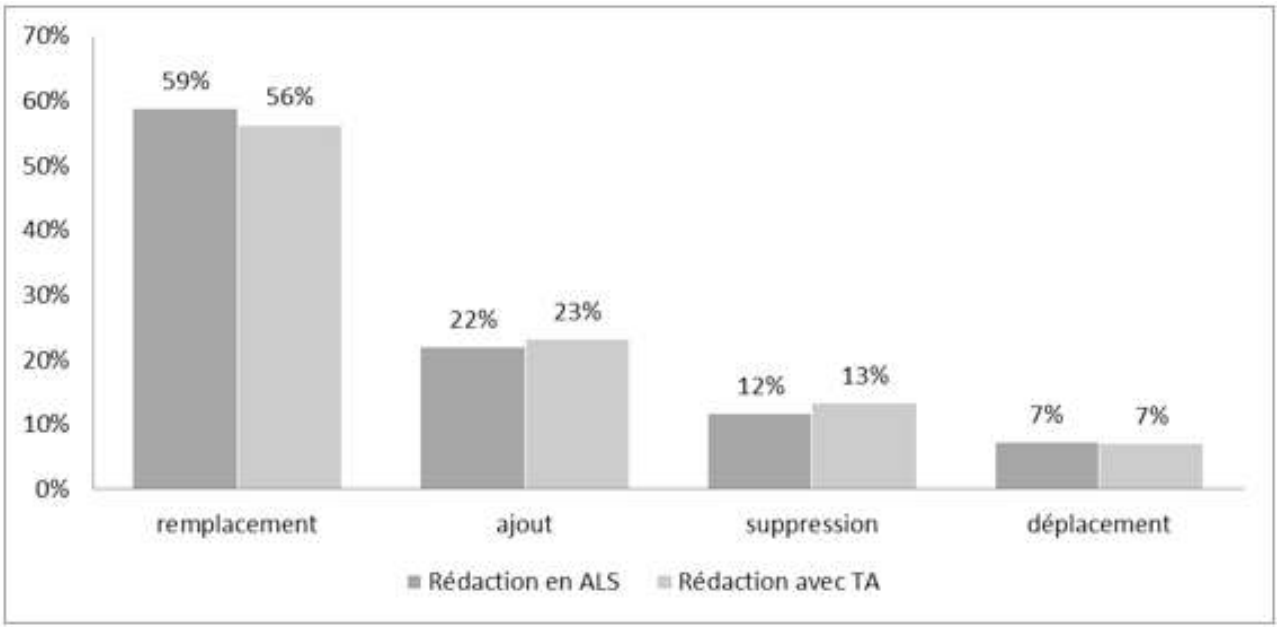

En premier lieu, on constate dans la figure 3 que les distributions des types d'opérations sont relativement similaires dans les deux situations, puisqu'on y observe à peu près les mêmes proportions de remplacements, d'ajouts, de suppressions et de déplacements.

Figure 4. Distribution des modifications par type d'unité linguistique

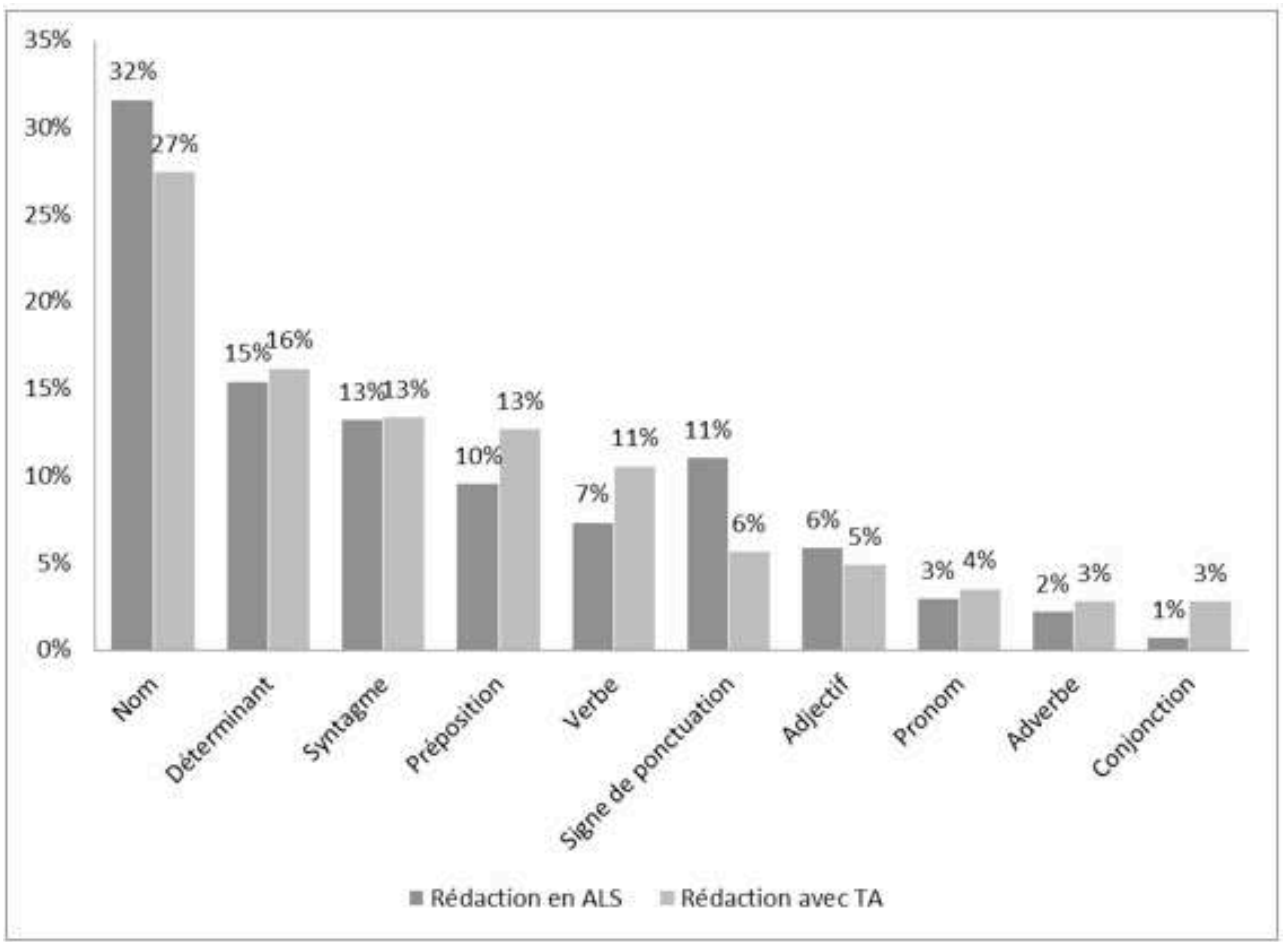

41 La figure 4 présente la distribution des modifications en fonction du type d'unité linguistique affectée par une modification. Si l'on se concentre sur les écarts de $3 \%$ et plus, on constate que les modifications de la réviseure professionnelle concernent moins souvent les noms et les signes de ponctuation dans les parties où la TA a été utilisée. En revanche, on observe le phénomène inverse pour les prépositions et les 
verbes, c'est-à-dire qu'il y a plus de modifications dans les textes produits avec l'aide de la TA que dans ceux rédigés en ALS.

La distribution des modifications selon les dimensions linguistiques est présentée dans la figure 5.

Figure 5. Distribution des modifications par dimension linguistique

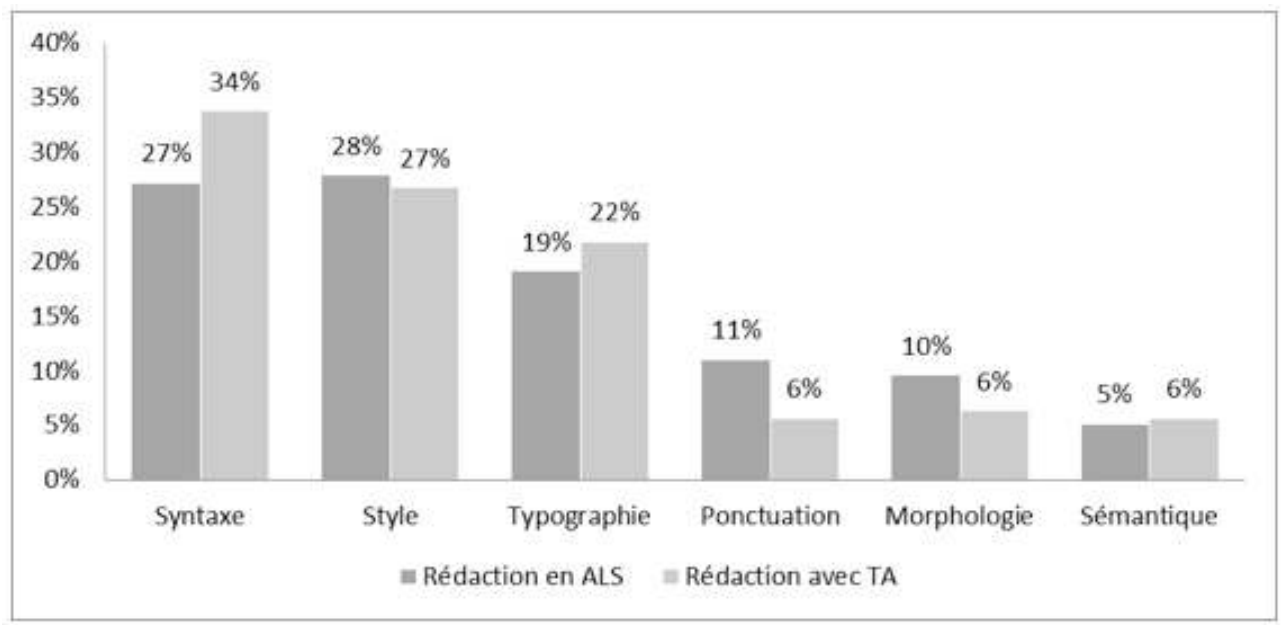

On remarque que, dans les textes produits avec la TA, les modifications affectant la syntaxe et la typographie représentent une plus grande proportion que dans les textes rédigés en ALS, alors que les modifications affectant la morphologie et la ponctuation sont en moindre proportion. Certains écarts demeurent tout de même faibles et il est loin d'être certain qu'ils soient significatifs.

Il est intéressant de noter que les modifications de nature typographique représentent une importante proportion des modifications apportées par la réviseure, que ce soit dans l'une ou l'autre des situations. En pratique, une forte proportion de ces modifications a trait à l'usage des majuscules dans les titres en anglais. Il faut toutefois admettre que cet aspect est moins problématique que d'autres pour la compréhension d'un texte.

Cette analyse nous permet de répondre à notre deuxième question de recherche, qui se formulait comme suit : les modifications linguistiques sont-elles du même type dans les deux situations? La réponse varie selon l'aspect analysé. En ce qui concerne les types d'opération, nous n'avons observé aucune différence digne de mention. Quant aux types d'unité linguistique, des différences ont été observées : les textes rédigés avec la TA ont fait l'objet de plus de modifications sur les verbes et les prépositions, en comparaison avec les textes rédigés en ALS. De plus, l'analyse des dimensions linguistiques indique que la réviseure a effectué davantage de modifications relevant de la syntaxe dans les textes rédigés avec la TA.

Dans le reste de cette section, nous abordons les facteurs qui auraient pu influencer ces résultats, en commençant par la langue maternelle des participants. La figure 6 présente les taux de modification pour chaque participant, dans les deux situations. 
Figure 6. Taux de modification en pourcentage pour chaque participant

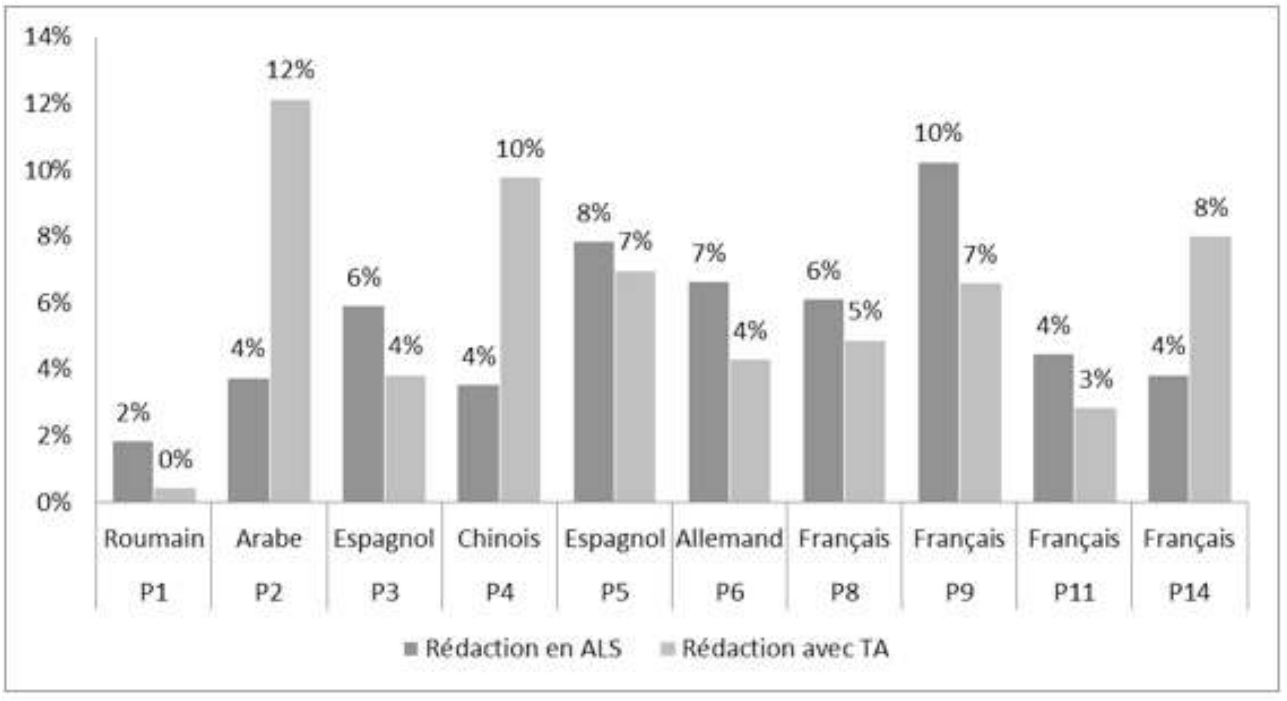

Comme on peut le voir dans la figure 6, les taux de modification varient considérablement d'un texte à l'autre, de même que d'un participant à l'autre. Pour les participants P2, P4 et P14, les textes produits avec la TA présentent des taux de modification supérieurs à ceux des textes rédigés en ALS, ce qui suggère que les textes produits à l'aide de la TA étaient de moins bonne qualité dans ces trois cas. Dans les sept autres cas, on observe le phénomène inverse, c'est-à-dire que les textes produits avec la TA présentent des taux de modification inférieurs à ceux des textes rédigés en ALS, quoique les différences entre les deux situations soient généralement moins prononcées. Nous croyons néanmoins que ces résultats laissent entendre qu'il existe des situations pour lesquelles la rédaction scientifique assistée par la TA présenterait des avantages, alors que pour d'autres personnes, cette manière de produire des textes scientifiques ne serait pas appropriée.

L'on ne peut passer sous silence que les plus grands écarts de qualité en défaveur de la TA se situent chez les participants arabophone (P2) et sinophone (P4). Dans ces deux cas, les taux de modification dans les textes produits avec la TA sont respectivement de 12 et de 10 , alors que le taux dans les textes rédigés en ALS est de 4 . Il est également à noter que 12 et 10 sont parmi les taux les plus élevés, toutes situations confondues. Comment expliquer ces résultats? Nous excluons que le niveau de compétence en anglais soit ici la principale cause, puisque le taux de modification dans les textes rédigés en ALS est de 4 dans les deux cas, une valeur parmi les plus basses dans notre étude. La qualité linguistique des textes rédigés en ALS par P2 et P4 était donc plus élevée que dans bien d'autres textes de notre corpus. Le niveau de compétence en anglais, tel que déclaré par les participants, va dans le même sens: le participant arabophone se perçoit comme un locuteur avancé de l'anglais, alors que le participant sinophone se décrit comme un locuteur intermédiaire.

Ce raisonnement nous amène à poser l'hypothèse que la qualité linguistique des textes anglais générés par le système de TA serait en cause pour ces deux langues. En effet, les systèmes de TA traduisent généralement plus aisément entre des paires de langues qui sont apparentées (Koehn et alii 2009). La TA statistique, celle que nous avons utilisée, est typiquement beaucoup plus difficile du chinois et de l'arabe vers l'anglais que pour des langues européennes telles que le français ou l'espagnol. Suivant cette hypothèse, 
nous pouvons imaginer qu'en deçà d'un certain niveau de qualité, le nombre d'erreurs à repérer et à corriger devient tel que le résultat final s'en trouve affecté, quel que soit le niveau de compétence linguistique de l'utilisateur.

Bien que cette étude exploratoire n'ait pas comme objectif d'analyser les modifications effectuées par les participants, nous avons tout de même calculé le pourcentage de mots modifiés par les participants dans les parties où la TA a été utilisée, autrement dit : le pourcentage de mots post-édités. Ces résultats sont présentés dans la figure 7.

Figure 7. Pourcentage des mots post-édités par les participants dans les textes rédigés avec l'aide de la TA

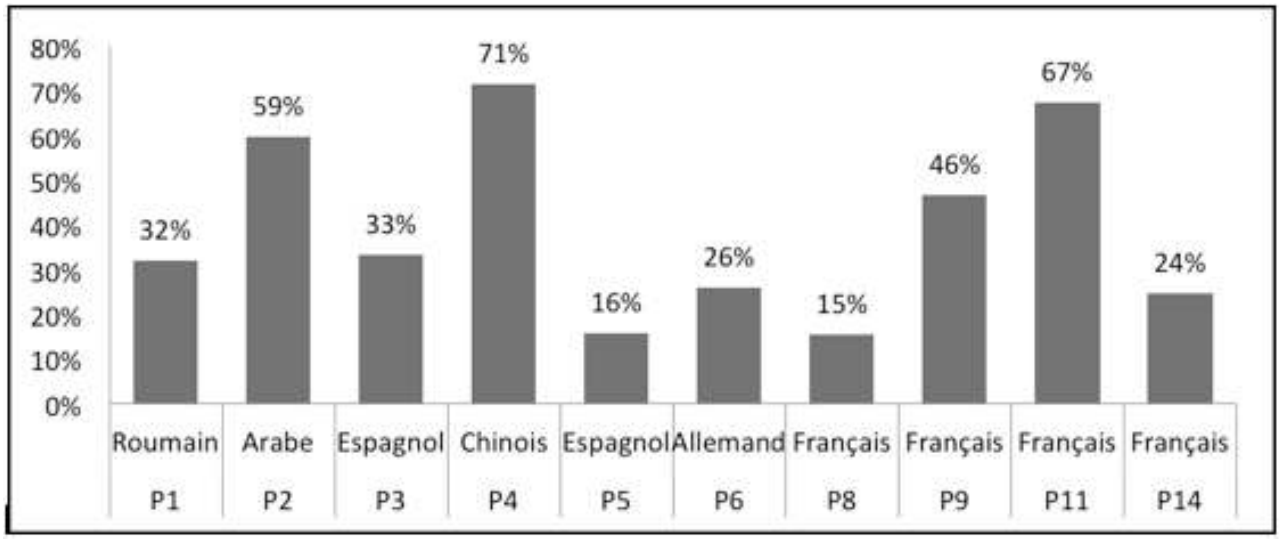

51 Comme on peut le constater, les participants arabophone et sinophone sont parmi ceux qui ont effectué le plus de modifications dans les textes produits avec la TA. Cette observation pourrait suggérer que ces deux participants étaient confrontés à des traductions dont la qualité était particulièrement médiocre. Bien entendu, nos données sont en quantité insuffisante pour quelque généralisation que ce soit, mais elles pourraient suggérer que, pour certaines paires de langues, le fait d'apporter de nombreuses modifications n'entraînera pas nécessairement un texte de qualité acceptable. À un extrême, un chercheur possédant une excellente maîtrise de l'anglais de spécialité n'aura pas énormément à gagner à utiliser la TA. Certaines des erreurs introduites par la TA pourraient même échapper à sa vigilance et affecter négativement la qualité du texte. À l'autre extrême, un chercheur qui ne maîtrise pas ou peu l'anglais de spécialité ne sera vraisemblablement pas en mesure de détecter les erreurs de traduction, encore moins de les corriger. Toutefois, entre ces deux extrêmes, il existe possiblement des profils d'utilisateurs pour lesquels la TA pourrait s'avérer un outil avantageux. Dans la conclusion, nous présentons justement les pistes de recherche que nous a inspirées cette étude exploratoire.

\section{Conclusion}

L'objectif de cet article était d'explorer la pertinence d'utiliser la TA comme outil d'aide à la rédaction d'articles scientifiques en anglais langue seconde. Pour ce faire, nous avons demandé à dix chercheurs de rédiger chacun deux textes : un premier texte rédigé directement en ALS et un second texte rédigé en langue maternelle, traduit automatiquement et post-édité. La qualité linguistique des textes ainsi produits a été évaluée grâce à une analyse des modifications effectuées par une réviseure professionnelle, laquelle n'était pas au courant des deux contextes de rédaction. Dans la 
présentation des résultats, nous avons vu que le taux de modification - c'est-à-dire le nombre de modifications par 100 mots - était de 5 pour les textes rédigés en ALS et de 6 pour les textes produits avec l'aide de la TA. Cette légère différence nous a amenés à conclure que la qualité linguistique était sensiblement la même dans les deux types de textes. Par conséquent, si un chercheur préférait rédiger dans sa langue maternelle plutôt que directement en ALS, cette option ne détériorerait pas la qualité du texte, du moins dans certaines situations.

Alors que les opérations effectuées par la réviseure professionnelle étaient sensiblement les mêmes dans les deux situations, nous avons observé certaines différences dans les unités linguistiques et dans les dimensions touchées. Notamment, la réviseure a effectué plus de modifications touchant les verbes et les prépositions dans les textes produits avec l'aide de la TA que dans ceux rédigés en ALS. En ce qui concerne les dimensions linguistiques, les textes rédigés avec la TA ont entraîné plus de modifications syntaxiques. Ainsi, la question qui en découle naturellement est celle de savoir si des erreurs spécifiques se répètent. Le cas échéant, il serait intéressant d'effectuer une recherche dans laquelle des consignes précises de post-édition seraient données aux chercheurs. Par la suite, l'on pourrait mesurer la qualité linguistique obtenue dans cette nouvelle situation. L'on pourrait aussi concevoir une recherche où les chercheurs reçoivent une formation sur la post-édition, comme dans l'étude d'E. M. O'Neill (2012) avec des apprenants langue seconde.

En outre, nous croyons qu'il serait pertinent de tenir compte, dans une recherche ultérieure, de l'importance relative des modifications linguistiques dans l'évaluation de la qualité des textes. Par exemple, Hanna Y. Touchie (1986) établit une distinction entre les "erreurs globales", qui peuvent toucher notamment l'ordre des mots dans une phrase (donc la syntaxe), et les "erreurs locales", qui concernent les accords nominaux et verbaux, l'utilisation des déterminants, des prépositions et des auxiliaires, entre autres. Ajoutons à cette considération que la qualité du contenu des textes devrait elle aussi, idéalement, faire l'objet d'une analyse. Le contenu pourrait être évalué par des chercheurs du même domaine, par exemple.

De plus, si nous sommes convaincus qu'il en vaille la peine de poursuivre l'exploration de l'utilité de la TA pour la rédaction scientifique en ALS, nous sommes tout aussi convaincus qu'il faudrait contrôler la variable langagière dans les recherches ultérieures. En effet, il est aisé d'admettre que la TA statistique de Google a donné de moins bons résultats pour le chinois et pour l'arabe, notamment, lorsque les textes étaient traduits vers l'anglais. Toutefois, les bonds prodigieux que fait la TA neuronale en ce moment nous permettent de croire que la méthode proposée dans cet article, à savoir l'utilisation de la TA comme aide à la rédaction scientifique en ALS, ne pourra que donner de meilleurs résultats dans le futur. 


\section{BIBLIOGRAPHIE}

BAHADUR, Promila \& Durg SINGH CHAUDAN. 2014. « Machine translation : A journey ». Actes de la Science and Information Conference, 187-195.

BENNETT, Karen. 2013. « English as a lingua franca in academia ». The Interpreter and Translator Trainer 7/2, 169-193.

BENNETT, Karen. 2014a. " The political and economic infrastructure of academic practice: the 'semi-periphery' as a category for social and linguistic analysis ». In BENNETT, K. (dir.), The Semiperiphery of Academic Writing: Discourses, communities and practices. Londres : Palgrave Macmillan, $1-12$.

BENNETT, Karen. 2014b. « Conclusion: Combating the centripetal pull in academic writing ». In BENNETT, K. (dir.), The Semi-periphery of Academic Writing: Discourses, communities and practices. Londres : Palgrave Macmillan, 240-246.

BENNETT, Karen. 2015. « Towards an epistemological monoculture: mechanisms of epistemicide in European research publication ». In PLO, R. \& C. PÉREZ-LLANTADA (dir.), English as an Academic and Research Language (English in Europe Vol. 2). De Gruyter Mouton : Berlin, 9-35.

BENFIELD, John R. \& Christine B. FEAK. 2006. « How authors can cope with the burden of English as an international language ». Chest $129 / 6,1728-1730$.

BREUER, Esther Odilia. 2015. First Language Versus Foreign Language. Fluency, errors and revision processes in foreign language academic writing. Frankfurt am Main : Peter Lang Edition.

BURGESS, Sally, Maria LLUÏSA GEA-VALOR, Ana I. MORENO \& Jesús J. REY-ROCHA. 2014. « Affordances and constraints on research publication: A comparative study of the language choices of Spanish historians and psychologists ». Journal for English for Academic Purposes 14, 72-83.

DEPALMA, Donald A., Hélène PIELMEIER, Robert G. STEWART \& Vijayalaxmi HEDGE. 2013. « The language service market: 2013 », Common Sense Advisory Report.

EBRAHIM, Sara, Doaa HEGAZY, Mostafa G. M. MOSTAFA \& Samhaa R. el-BELTAGY. 2015. « English-Arabic statistical machine translation: State of the art ». Lectures Notes in Computer Science 9041, 520-533. FERNÁNDEZ POLO, Francisco JAVIER \& Mario CAL VARELA. 2009. « English for research purposes at the University of Santiago de Compostela: a survey ». Journal of English for Academic Purposes 8/3, 152 164.

GARCIA, Ignacio \& María Isabel PENA. 2011. « Machine translation-assisted language learning: Writing for beginners ». Computer Assisted Language Learning 24/5, 471-487. doi: 10.1080/09588221.2011.582687.

GRAHAM, Mark, S. A. HALE \& M. STEPHENS. 2011. Geographies of the World's Knowledge. Londres : Convoco! Edition.

HANAUER, David I. \& Karen ENGLANDER. 2011. « Quantifying the burden of writing research articles in a second language: Data from Mexican scientists ». Written Communication 28/4, 403-416.

HU, Chang, Benjamin B. BEDERSON, Philip RESNIK \& Yakiv KRONROD. 2011. « MonoTrans2: A new human computation system to support monolingual translation ». Proceedings of the SIG-CHI Conference on Human Factors in Computing Systems, 1133-1136. 
IKES, Melida J. \& Stephen F. GAMBESCIA. 2011. « Abstract art: How to write competitive conference and journal abstracts ». Health Promotion Practice 12, 493-496.

KOEHN, Philipp, Alexandra BIRCH \& Ralf STEINBERGER. 2009. « 462 Machine Translation Systems for Europe ». Actes du MT Summit XII, consulté le 22 mars 2017 <http://www.mt-archive.info/ MTS-2009-Koehn-1.pdf>.

LAFLAMME, Caroline. 2009. «Les modifications lexicales apportées par les réviseurs professionnels dans leur tâche de révision : du problème à la solution ». Thèse de doctorat inédite, Université Laval, Québec, Canada.

LILLIS, Teresa \& Mary Jane CURRY. 2010. Academic Writing in a Global Context: The politics and practices of publishing in English. Londres \& New York : Routledge.

LÓPEZ-NAVARRO, Irene, Ana I. MORENO, Miguel Angel QUINTANILLA \& Jesús REY-ROCHA. 2015. « Why do I publish research articles in English instead of my own language? Differences in Spanish researchers' motivations across scientific domains ». Scientometrics 103, 939-976.

MARTíN, Pedro, Jesús REY-ROCHA, Sally BURGESS \& Ana I. MORENO. 2014. «Publishing research in English-language journals: Attitudes, strategies and difficulties of multilingual scholars of medicine ». Journal of English for Academic Purposes 16, 57-67.

MASSARDO, Isabella, Jaap VAN DER MEER, Sharon o'BRIEN, Fred HOLLYWOOD, Nora ARANBERRI \& Katrin DRESCHER. 2010. « MT post-editing guidelines ». Translation Automation User Society (TAUS), consulté le 16 juin 2017 <https://www.taus.net/academy/best-practices/postedit-best-practices/machinetranslation-post-editing-guidelines>.

MITCHELL, Linda, Sharon O'BRIEN \& Johann ROTURIER. 2014. « Quality evaluation in community postediting ». Machine Translation 28/3-4, 237-262.

MOSSOP, Brian. 2014. Revising and Editing for Translators. Oxon \& New York : Routledge.

NIÑo, Ana. 2008. « Evaluating the use of machine translation post-editing in the foreign language class ». Computer Assisted Language Learning 21/1, 29-49.

O'BRIEN, Sharon \& Michel SIMARD (dir.). 2014. « Special issue on post-editing ». Machine Translation $28,159-329$.

O'BRIEN, Sharon, Michel SIMARD \& Marie-Josée GOULET. À paraître. « Machine translation and selfpost-editing for academic writing support: Quality explorations ». In J. MOORKENS, S. CASTILHO, S. DOHERTY \& F. GASPARI (dir.), Human and Machine Tranlsation Quality and Evaluation. Springer.

O'NEILL, Errol Marinus. 2012. " The effect of online translators on L2 writing in French ». Thèse de doctorat inédite, University of Illinois at Urbana-Champaign, États-Unis, consulté le 6 juillet 2016 <http://hdl.handle.net/2142/34317>.

SÁNCHEZ-GIJÓN, Pilar \& Olga TORRES-HOSTENCH. 2014. « MT post-editing into the mother tongue or into a foreign language? Spanish-to-English MT translation output post-edited by translation trainees ». Proceedings of the 11th Conference of the Association for Machine Translation in the Americas. <http://www.amtaweb.org/AMT>.

SNOVER, Matthew, Bonnie DORR, Richard SCHWARTZ, Linnea MICCIULLA \& John MAKHOUL. 2006. «A study of translation edit rate with targeted human annotation ». Proceedings of the 7th Conference of the Association for Machine Translation in the Americas, 223-231.

TOUCHIE, Hanna Y. 1986. « Second language learning errors: Their types, causes and treatment ». JALT Journal 8/1, 75-80. 
TUROVSKY, Barak. 2016. « Found in translation: More accurate, fluent sentences in Google

Translate ». Google Blog, consulté le 5 juin 2017 <http://hdl.handle.net/2142/34317>.

VAN WAES, Luuk \& Mariëlle LEIJTEN. 2015. «Fluency in writing: A multidimensional perspective on writing fluency applied to L1 and L2 ». Computers and Composition 38, 79-95.

\section{NOTES}

1. Cette recherche a été en partie subventionnée par ADAPT Research Centre for Digital Content Technology, lequel est financé par Science Foundation Ireland Research Centres Programme (Subvention 13/RC/2106) et par European Regional Development Fund.

2. L'expérience dont il est question dans cet article est également présentée dans un chapitre de livre à paraitre en 2018 (O'Brien et alli). Dans ce dernier, toutefois, nous étudions d'autres aspects que la qualité linguistique, notamment le temps requis afin de rédiger et de post-éditer les textes. 3. Comme nous le préciserons plus loin dans l'article, nous utilisons le terme post-édition afin de désigner l'activité de révision d'un texte traduit automatiquement.

4. L'article d'A. Niño (2008) ne précise pas qui a corrigé les textes.

5. <http://tradukka.com>

6. <<http://freetranslation.com>

7. Ce dernier participant ne correspond pas exactement à nos critères de départ. Nous l'avons tout de même inclus dans l'expérience afin de pallier la difficulté de recruter des participants.

8. <http://www.granddictionnaire.com>

9. $<$ http://www.word reference.com>

10. <https://translate.google.com>

11. <http://www.systran.fr/lp/traduction-en-ligne/>

12. <http://www.reverso.net/text_translation.aspx?lang=FR>

13. <https://www.bing.com/translator>

\section{RÉSUMÉS}

Nous explorons dans cet article la pertinence d'utiliser la traduction automatique (TA) comme outil d'aide à la rédaction scientifique en anglais langue seconde (ALS). L'expérience, à laquelle ont participé dix chercheurs, comprenait deux tâches: la rédaction d'un texte en ALS et la rédaction d'un texte en langue maternelle. Ce dernier a ensuite été traduit automatiquement et post-édité par le participant. Par la suite, nous avons évalué la qualité linguistique des textes produits dans les deux situations, à partir d'une analyse des modifications linguistiques effectuées par une réviseure professionnelle. Selon nos résultats, la qualité linguistique des textes est sensiblement la même dans les deux situations, tandis que des différences apparaissent dans les dimensions linguistiques et dans les types d'unités touchées. L'article se termine par une réflexion sur les facteurs pouvant influencer ces résultats. Nous concluons en réitérant la pertinence d'étudier l'utilisation de la TA pour la rédaction d'articles en anglais de spécialité.

In this paper, we investigate the use of machine translation (MT) as an aid for writing academic texts in English as a second language (ESL). We report on an experiment, which involved ten 
researchers and included two tasks: writing a text in ESL and writing a text in the mother tongue. The second text was subsequently machine translated and post-edited by each participant. We then analysed the types of edits made by a professional reviser and used our analysis to evaluate the linguistic quality of the texts produced in both conditions. According to our results, the linguistic quality of the texts is equivalent in both conditions, whereas some differences appear in the linguistic dimensions and in the types of units affected by the edits. The article ends with a reflection on the factors that could have influenced the results. We conclude by reaffirming the relevance of studying the use of MT in writing in English for specific purposes.

\section{INDEX}

Keywords : academic writing, English as a second language, machine translation, post-editing, writing in English as a second language

Mots-clés : anglais langue seconde, post-édition, rédaction en anglais langue seconde, rédaction scientifique, traduction automatique

\section{AUTEURS}

\section{MARIE-JOSÉE GOULET}

Marie-Josée Goulet est professeur au département d'études langagières de l'Université du Québec en Outaouais. Elle enseigne la rédaction professionnelle générale, de même que la rédaction technique et scientifique. Ses recherches portent sur les outils informatiques dédiés à la rédaction, sur l'utilisation de la traduction automatique dans des contextes de rédaction et sur les compétences numériques dans les métiers de la communication.marie-josee.goulet@uqo.ca

\section{MICHEL SIMARD}

Michel Simard est chercheur au Conseil national de recherches Canada (CNRC), au sein de l'équipe Traitement de textes multilingues. Il œuvre depuis plus de 25 ans dans le domaine de la traduction automatique. Ses recherches portent plus particulièrement sur les nouvelles applications des outils de traduction automatique. michel.simard@cnrc-nrc.gc.ca

\section{CARLA PARRA ESCARTÍN}

Carla Parra Escartín est chercheur postdoctoral Marie Skłodowska-Curie au centre de recherche ADAPT à Dublin City University. Ses recherches actuelles portent sur l'impact de la traduction automatique dans les flots de travail en traduction professionnelle, de même que dans d'autres contextes d'utilisation. Elle s'intéresse en outre aux outils de traduction, à la qualité de la traduction, et aux considérations éthiques et légales dans les activités de recherche. carla.parra@adaptcentre.ie

\section{SHARON O'BRIEN}

Sharon O'Brien est professeur de traduction et de technologies langagières à la School of Applied Language and Intercultural Studies de Dublin City University. Ses recherches portent sur l'interaction entre les traducteurs et les technologies, les aspects cognitifs de la traduction, les méthodes de recherche (suivi oculaire et enregistrement du clavier), la localisation et la création de contenu. Elle travaille à titre de chercheur subventionné au centre interinstitutionnel ADAPT. sharon.obrien@dcu.ie 